\title{
The Global Attractors and Their Hausdorff and Fractal Dimensions Estimation for the Higher-Order Nonlinear Kirchhoff-Type Equation with Strong Linear Damping
}

\author{
Yunlong Gao, Yuting Sun, Guoguang Lin \\ Department of Mathematics, Yunnan University, Kunming, China \\ Email: gy10813101x@163.com,931149783@qq.com, gglin@ynu.edu.cn
}

How to cite this paper: Gao, Y.L., Sun, Y.T. and Lin, G.G. (2016) The Global Attractors and Their Hausdorff and Fractal Dimensions Estimation for the Higher-Order Nonlinear Kirchhoff-Type Equation with Strong Linear Damping. International Journal of Modern Nonlinear Theory and Application, 5, 185-202.

http://dx.doi.org/10.4236/ijmnta.2016.54018

Received: October 10, 2016

Accepted: November 20, 2016

Published: November 23, 2016

Copyright $\odot 2016$ by authors and Scientific Research Publishing Inc. This work is licensed under the Creative Commons Attribution International License (CC BY 4.0).

http://creativecommons.org/licenses/by/4.0/

\begin{abstract}
In this paper, we study the longtime behavior of solution to the initial boundary value problem for a class of strongly damped Higher-order Kirchhoff type equations: $u_{t t}+(-\Delta)^{m} u_{t}+\left\|D^{m} u\right\|^{2 q}(-\Delta)^{m} u+g(u)=f(x)$. At first, we prove the existence and uniqueness of the solution by priori estimation and the Galerkin method. Then, we obtain to the existence of the global attractor. At last, we consider that the estimation of the upper bounds of Hausdorff and fractal dimensions for the global attractors are obtained.
\end{abstract}

\section{Keywords}

Nonlinear Higher-Order Kirchhoff Type Equation, The Existence and Uniqueness, The Global Attractors, Hausdorff Dimensions, Fractal Dimensions

\section{Introduction}

In this paper, we are concerned with the existence of global attractor and Hausdorff and Fractal dimensions estimation for the following nonlinear Higher-order Kirchhoff-type equations:

$$
\begin{gathered}
u_{t t}+(-\Delta)^{m} u_{t}+\left\|D^{m} u\right\|^{2 q}(-\Delta)^{m} u+g(u)=f(x),(x, t) \in \Omega \times[0,+\infty), \\
u(x, 0)=u_{0}(x), u_{t}(x, 0)=u_{1}(x), x \in \Omega, \\
u(x, t)=0, \frac{\partial^{i} u}{\partial v^{i}}=0, i=1, \cdots, m-1, x \in \partial \Omega, t \in(0,+\infty),
\end{gathered}
$$


where $m>1$ is an integer constant, and $q>0$ is a positive constant. Moreover, $\Omega$ is a bounded domain in $R^{n}$ with the smooth boundary $\partial \Omega$ and $v$ is the unit outward normal on $\partial \Omega . g(u)$ is a nonlinear function specified later.

Recently, Marina Ghisi and Massimo Gobbino [1] studied spectral gap global solutions for degenerate Kirchhoff equations. Given a continuous function $m:[0,+\infty) \rightarrow[0,+\infty)$, they consider the Cauchy problem:

$$
\begin{gathered}
u_{t t}(t, x)+m\left(\int_{\Omega}|\nabla u(t, x)|^{2} \mathrm{~d} x\right) \Delta u(t, x)=0, \forall(t, x) \in \Omega \times[0, T), \\
u(0)=u_{0}, u_{t}(0)=u_{1},
\end{gathered}
$$

where $\Omega \subseteq R^{n}$ is an open set and $\nabla u$ and $\Delta u$ denote the gradient and the Laplacian of $u$ with respect to the space variables. They prove that for such initial data $\left(u_{0}, u_{1}\right)$ there exist two pairs of initial data $\left(\bar{u}_{0}, \bar{u}_{1}\right),\left(\hat{u}_{0}, \hat{u}_{1}\right)$ for which the solution is global, and such that $u_{0}=\bar{u}_{0}+\hat{u}_{0}, u_{1}=\bar{u}_{1}+\hat{u}_{1}$.

Yang Zhijian, Ding Pengyan and Lei Li [2] studied Longtime dynamics of the Kirchhoff equations with fractional damping and supercritical nonlinearity:

$$
\begin{gathered}
u_{t t}-M\left(\|\nabla u\|^{2}\right) \Delta u+(-\Delta)^{\alpha} u_{t}+f(u)=g(x), x \in \Omega, t>0, \\
\left.u\right|_{\partial \Omega}=0, u(x, 0)=u_{0}(x), u_{t}(x, 0)=u_{1}(x),
\end{gathered}
$$

where $\alpha \in\left(\frac{1}{2}, 1\right), \Omega$ is a bounded domain $R_{N}$ with the smooth boundary $\partial \Omega$, and the nonlinearity $f(u)$ and external force term $g$ will be specified. The main results are focused on the relationships among the growth exponent $p$ of the nonlinearity $f(u)$ and well-posedness. They show that (i) even if $p$ is up to the supercritical range, that is, $1 \leq p<\frac{N+4 \alpha}{(N-4 \alpha)^{+}}$, the well-posedness and the longtime behavior of the solutions of the equation are of the characters of the parabolic equation; (ii) when $\frac{N+4 \alpha}{(N-4 \alpha)^{+}} \leq p<\frac{N+4}{(N-4)^{+}}$, the corresponding subclass $\mathrm{G}$ of the limit solutions exists and possesses a weak global attractor.

Yang Zhijian, Ding Pengyan and Liu Zhiming [3] studied the Global attractor for the Kirchhoff type equations with strong nonlinear damping and supercritical nonlinearity:

$$
\begin{gathered}
u_{t t}-\sigma\left(\|\Delta u\|^{2}\right) \Delta u_{t}-\phi\left(\|\Delta u\|^{2}\right) \Delta u+f(u)=h(x) \quad \text { in } \Omega \times \mathbb{R}^{+}, \\
\left.u(x, t)\right|_{\partial \Omega}=0, u(x, 0)=u_{0}(x), u_{t}(x, 0)=u_{1}(x), \quad x \in \Omega .
\end{gathered}
$$

where $\Omega$ is a bounded domain in $R^{N}$ with the smooth boundary $\partial \Omega, \sigma(s), \phi(s)$ and $f(s)$ are nonlinear functions, and $h(x)$ is an external force term. They prove that in strictly positive stiffness factors and supercritical nonlinearity case, there exists a global finite-dimensional attractor in the natural energy space endowed with strong topology.

Li Fucai [4] studied the global existence and blow-up of solutions for a higher-order 
nonlinear Kirchhoff-type hyperbolic equation:

$$
\begin{gathered}
u_{t t}+\left(\int_{\Omega}\left|D^{m} u\right|^{2} \mathrm{~d} x\right)^{q}(-\Delta)^{m} u+u_{t}\left|u_{t}\right|^{r}=|u|^{p} u, x \in \Omega, t>0, \\
u(x, t)=0, \frac{\partial^{i} u}{\partial v^{i}}=0, i=1,2, \cdots, m-1, x \in \partial \Omega, t>0, \\
u(x, 0)=u_{0}(x), u_{t}(x, 0)=u_{1}(x),
\end{gathered}
$$

where $m \geq 1, p, q, r \geq 0, \Omega$ is a bounded domain $R_{n}$, with a smooth boundary $\partial \Omega$ and a unit outer normal $v$. Setting $E(t)=\frac{1}{2}\left\|u_{t}\right\|_{2}^{2}+\frac{1}{2(q+1)}\left\|D^{m} u\right\|_{2}^{2(q+1)}-\frac{1}{p+2}\|u\|_{p+2}^{p+2}$.
Assume that $\mathrm{p}$ satisfies the condition:

$$
p \leq \frac{2}{N-2 m} \text {, for } N>2 m ; p>0 \text {, for } N \leq 2 m \text {. }
$$

Their main results are the two theorems:

Theorem 1. Suppose that $p \leq r$ and condition (1.13) holds. Then for any initial data $\left(u_{0}, u_{1}\right) \in H^{2 m}(\Omega) \cap H_{0}^{m}(\Omega) \times H_{0}^{m}(\Omega)$, the solution of (1.10) - (1.12) exists globally.

Theorem 2. Suppose that $p>\max \{r, 2 q\}$ and condition (1.12) holds. Then for any initial data $\left(u_{0}, u_{1}\right) \in H^{2 m}(\Omega) \cap H_{0}^{m}(\Omega) \times H_{0}^{m}(\Omega)$, the solution of (1.10) - (1.12) blows up at finite time in $L_{p+2}$ norm provided that $E(0)<0$.

Li Yan [5] studied The Asymptotic Behavior of Solutions for a Nonlinear Higher Order Kirchhoff Type Equation:

$$
\begin{gathered}
u_{t t}+\left(\int_{\Omega}\left|D^{m} u\right|^{2} \mathrm{~d} x\right)^{q}(-\Delta)^{m} u+\beta u_{t}+g(u)=0, \quad \text { in } Q=\Omega \times(0,+\infty), \\
u(x, t)=0, \frac{\partial^{i} u}{\partial v^{i}}=0, i=1,2, \cdots, m-1, \quad \text { on } \Sigma=\Gamma \times(0,+\infty), \\
u(x, 0)=u_{0}(x), u_{t}(x, 0)=u_{1}(x), \quad \text { in } x \in \Omega,
\end{gathered}
$$

where $\Omega$ is an open bounded set of $R^{n}(n \geq 1)$ with smooth boundary $\Gamma$ and the unit normal vector. The function $g \in C^{1}$ satisfies the following conditions:

$$
\begin{gathered}
\lim _{|s| \rightarrow \infty} \inf \frac{G(s)}{s^{2}} \geq 0, G(s)=\int_{0}^{s} g(r) \mathrm{d} r ; \\
\liminf _{|s| \rightarrow \infty} \frac{\left|g^{\prime}(s)\right|}{|s|^{\gamma}}=0,
\end{gathered}
$$

where $0 \leq \gamma<+\infty(n=1,2), 0 \leq \gamma<2(n=3), \gamma=0(n \geq 4)$. Furthermore, there exists $C_{1}>0$ such that

$$
\lim _{|s| \rightarrow \infty} \inf \frac{s g(s)-C_{1} G(s)}{s^{2}} \geq 0 .
$$

At last, Li Yan studied the asymptotic behavior of solutions for problem (1.14) (1.16).

For the most of the scholars represented by Yang Zhijian have studied all kinds of low order Kirchhoff equations and only a small number of scholars have studied the 
blow-up and asymptotic behavior of solutions for higher-order Kirchhoff equation. So, in this context, we study the high-order Kirchhoff equation is very meaningful. In order to study the high-order nonlinear Kirchhoff equation with the damping term, we borrow some of Li Yan's [5] partial assumptions (2.1) - (2.3) for the nonlinear term $g$ in the equation. In order to prove that the lemma 1, we have improved the results from assumptions (2.1) - (2.3) such that $0<C_{2} \leq 1$. Then, under all assumptions, we prove that the equation has a unique smooth solution $\left(u, u_{t}\right) \in L^{\infty}\left((0,+\infty) ; H^{2 m}(\Omega) \times H_{0}^{m}(\Omega)\right)$ and obtain the solution semigroup $S(t): H^{2 m}(\Omega) \times H_{0}^{m}(\Omega) \rightarrow H^{2 m}(\Omega) \times H_{0}^{m}(\Omega)$ has global attractor $\mathcal{A}$. Finally, we prove the equation has finite Hausdorff dimensions and Fractal dimensions by reference to the literature [7].

For more related results we refer the reader to [6] [7] [8] [9] [10]. In order to make these equations more normal, in section 2 and in section 3, some assumptions, notations and the main results are stated. Under these assumptions, we prove the existence and uniqueness of solution, then we obtain the global attractors for the problems (1.1) (1.3). According to [6] [7] [8] [9] [10], in section 4, we consider that the global attractor of the above mentioned problems (1.1) - (1.3) has finite Hausdorff dimensions and fractal dimensions.

\section{Preliminaries}

For convenience, we denote the norm and scalar product in $L^{2}(\Omega)$ by $\|$.$\| and (.,$.$) ;$ $f=f(x), \quad L^{p}=L^{p}(\Omega), \quad H^{k}=H^{k}(\Omega), \quad H_{0}^{k}=H_{0}^{k}(\Omega),\|\cdot\|=\|\cdot\|_{L^{2}},\|\cdot\|_{p}=\|\cdot\|_{L^{p}}$.

According to [5], we present some assumptions and notations needed in the proof of our results. For this reason, we assume nonlinear term $g(u) \in C^{1}(\Omega)$ satisfies that $\left(\mathrm{H}_{1}\right)$ Setting $G(s)=\int_{0}^{s} g(r) \mathrm{d} r$, then

$$
\lim _{|s| \rightarrow \infty} \inf \frac{G(s)}{s^{2}} \geq 0
$$

$\left(\mathrm{H}_{2}\right)$ If

$$
\lim _{|s| \rightarrow \infty} \sup \frac{\left|g^{\prime}(s)\right|}{|s|^{r}}=0,
$$

where $0 \leq r<+\infty(n=1,2), 0 \leq r<2(n=3), r=0(n \geq 4)$.

$\left(\mathrm{H}_{3}\right)$ There exist constant $C_{0}>0$, such that

$$
\lim _{|s| \rightarrow \infty} \inf \frac{s g(s)-C_{0} G(s)}{s^{2}} \geq 0 .
$$

$\left(\mathrm{H}_{4}\right)$ There exist constant $C_{1}>0$, such that

$$
\begin{gathered}
|g(s)| \leq C_{1}\left(1+|s|^{p}\right), \\
\left|g^{\prime}(s)\right| \leq C_{1}\left(1+|s|^{p-1}\right),
\end{gathered}
$$

where $1 \leq p \leq \frac{n}{n-2 m}$; 
For every $\gamma>0$, by $\left(\mathrm{H}_{1}\right)-\left(\mathrm{H}_{3}\right)$ and apply Poincaré inequality, there exist constants $C(\gamma)>0$, such that

$$
\begin{gathered}
J(u)+\gamma\left\|D^{m} u\right\|^{2}+C(\gamma) \geq 0, \quad \forall u \in H^{m}(\Omega), \\
(g(u), u)-C_{2} J(u)+\gamma\left\|D^{m} u\right\|^{2}+C(\gamma) \geq 0, \quad \forall u \in H^{m}(\Omega),
\end{gathered}
$$

where $J(u)=\int_{\Omega} G(u) \mathrm{d} x, 0<C_{2} \leq 1$ is independent of $\gamma$.

Lemma 1. Assume $\left(\mathrm{H}_{1}\right)-\left(\mathrm{H}_{3}\right)$ hold, and $\left(u_{0}, u_{1}\right) \in H_{0}^{m}(\Omega) \times L^{2}(\Omega), f(x) \in L^{2}(\Omega)$. Then the solution $(u, v)$ of the problem (1.1) - (1.3) satisfies $(u, v) \in L^{\infty}\left((0,+\infty) ; H_{0}^{m}(\Omega) \times L^{2}(\Omega)\right)$, and

$$
\left\|D^{m} u\right\|^{2}+\|v\|^{2} \leq y(0) \mathrm{e}^{-C_{2} \varepsilon t}+\frac{\tilde{C}}{C_{2} \varepsilon}+\left(2^{q^{2}+2 q+1}-1\right) \frac{q}{q+1} .
$$

where $v=u_{t}+\varepsilon u, \quad 0<\varepsilon<\min \left\{\frac{\lambda_{1}^{m}}{1+2 \lambda_{1}^{m}}, \frac{\sqrt{1+4 \lambda_{1}^{m}}-1}{2}, \frac{\sqrt{\left(2+C_{2}\right)^{2}+16 \lambda_{1}^{m}}-2-C_{2}}{4}\right\}, \lambda_{1}$ is the first eigenvalue of $-\Delta$ in $H_{0}^{1}(\Omega)$, and

$$
\begin{aligned}
& y(0)=\left\|u_{1}+\varepsilon u_{0}\right\|^{2}-\varepsilon\left\|D^{m} u_{0}\right\|^{2}+\frac{1}{q+1}\left\|D^{m} u_{0}\right\|^{2 q+2}+\frac{q}{q+1}+2 J\left(u_{0}\right)+2 C\left(\gamma_{2}\right), \\
& \tilde{C}=\frac{1}{\varepsilon^{2}}\|f\|^{2}+2 \varepsilon C\left(\gamma_{1}\right)+q \varepsilon+\frac{q w}{q+1}+2 \varepsilon C_{2} C\left(\gamma_{2}\right), \quad \gamma_{1}=\frac{1}{2}-\frac{\varepsilon}{2 \lambda^{m}}-\varepsilon>0, \quad \gamma_{2}=\frac{1-\varepsilon}{2}>0,
\end{aligned}
$$
$w=\min \left\{2 \lambda_{1}^{m}-2 \varepsilon^{2}-2 \varepsilon,(q+1) \varepsilon\right\}$. Thus, there exists $E_{0}$ and $t_{0}=t_{0}(\Omega)>0$, such that

$$
\|(u, v)\|_{H_{0}^{m}(\Omega) \times L^{2}(\Omega)}^{2}=\left\|D^{m} u\right\|^{2}+\|v\|^{2} \leq E_{0}, \quad\left(t>t_{0}\right) .
$$

Proof. We take the scalar product in $L^{2}$ of equation (1.1) with $v=u_{t}+\varepsilon u$. Then

$$
\left(u_{t t}+(-\Delta)^{m} u_{t}+\left\|D^{m} u\right\|^{2 q}(-\Delta)^{m} u+g(u), v\right)=(f(x), v) .
$$

After a computation in (2.10), we have

$$
\begin{gathered}
\left(u_{t t}, v\right)=\frac{1}{2} \frac{\mathrm{d}}{\mathrm{d} t}\|v\|^{2}-\varepsilon\|v\|^{2}+\varepsilon^{2}(u, v), \\
\left((-\Delta)^{m} u_{t}, v\right)=-\frac{\varepsilon}{2} \frac{\mathrm{d}}{\mathrm{d} t}\left\|D^{m} u\right\|^{2}+\left\|D^{m} v\right\|^{2}-\varepsilon^{2}\left\|D^{m} u\right\|^{2} \\
\left(\left\|D^{m} u\right\|^{2 q}(-\Delta)^{m} u, v\right)=\frac{1}{2(q+1)} \frac{\mathrm{d}}{\mathrm{d} t}\left\|D^{m} u\right\|^{2 q+2}+\varepsilon\left\|D^{m} u\right\|^{2 q+2}, \\
(g(u), v)=\frac{\mathrm{d}}{\mathrm{d} t} J(u)+\varepsilon(g(u), u) .
\end{gathered}
$$

Collecting with (2.11) - (2.14), we obtain from (2.10) that

$$
\begin{aligned}
& \frac{1}{2} \frac{\mathrm{d}}{\mathrm{d} t}\left(\|v\|^{2}-\varepsilon\left\|D^{m} u\right\|^{2}+\frac{1}{q+1}\left\|D^{m} u\right\|^{2 q+2}+2 J(u)\right)-\varepsilon\|v\|^{2}+\varepsilon^{2}(u, v) \\
& +\left\|D^{m} v\right\|^{2}-\varepsilon^{2}\left\|D^{m} u\right\|^{2}+\varepsilon\left\|D^{m} u\right\|^{2 q+2}+\varepsilon(g(u), u)=(f(x), v) .
\end{aligned}
$$


Since $v=u_{t}+\varepsilon u$ and

$0<\varepsilon<\min \left\{\frac{\lambda_{1}^{m}}{1+2 \lambda_{1}^{m}}, \frac{\sqrt{1+4 \lambda_{1}^{m}}-1}{2}, \frac{\sqrt{\left(2+C_{2}\right)^{2}+16 \lambda_{1}^{m}}-2-C_{2}}{4}\right\}$, by using Hölder in-

equality Young's inequality and Poincaré inequality, we deal with the terms in (2.15) one by one as follow:

$$
\begin{gathered}
\varepsilon^{2}(u, v) \geq-\frac{\varepsilon^{2}}{2}\|u\|^{2}-\frac{\varepsilon^{2}}{2}\|v\|^{2} \geq-\frac{\varepsilon^{2}}{2 \lambda_{1}^{m}}\left\|D^{m} u\right\|^{2}-\frac{\varepsilon^{2}}{2}\|v\|^{2}, \\
\left\|D^{m} v\right\|^{2} \geq \lambda_{1}^{m}\|v\|^{2} .
\end{gathered}
$$

By (2.7), we can obtain

$$
\varepsilon(g(u), u) \geq C_{2} \varepsilon J(u)-\left(\frac{\varepsilon}{2}-\frac{\varepsilon^{2}}{2 \lambda_{1}^{m}}-\varepsilon^{2}\right)\left\|D^{m} u\right\|^{2}-\varepsilon C\left(\gamma_{1}\right),
$$

where $\gamma_{1}=\frac{1}{2}-\frac{\varepsilon}{2 \lambda_{1}^{m}}-\varepsilon>0$.

Because of $f(x) \in L^{2}(\Omega)$, we can obtain

$$
(f(x), v) \leq\|f\|\|v\| \leq \frac{\|f\|^{2}}{2 \varepsilon^{2}}+\frac{\varepsilon^{2}}{2}\|v\|^{2} .
$$

By (2.16) - (2.19), it follows from that

$$
\begin{aligned}
& \frac{\mathrm{d}}{\mathrm{d} t}\left(\|v\|^{2}-\varepsilon\left\|D^{m} u\right\|^{2}+\frac{1}{q+1}\left\|D^{m} u\right\|^{2 q+2}+2 J(u)\right)+\left(2 \lambda_{1}^{m}-2 \varepsilon^{2}-2 \varepsilon\right)\|v\|^{2} \\
& -\varepsilon\left\|D^{m} u\right\|^{2}+2 \varepsilon\left\|D^{m} u\right\|^{2 q+2}+2 C_{2} \varepsilon J(u) \leq \frac{1}{\varepsilon^{2}}\|f\|^{2}+2 \varepsilon C\left(\gamma_{1}\right) .
\end{aligned}
$$

By Young's inequality and $0<\varepsilon<\frac{\lambda_{1}^{m}}{1+2 \lambda_{1}^{m}}<1$, we have

$$
\begin{gathered}
\frac{1}{q+1}\left\|D^{m} u\right\|^{2 q+2}-\varepsilon\left\|D^{m} u\right\|^{2}+\frac{q}{q+1} \geq(1-\varepsilon)\left\|D^{m} u\right\|^{2} \geq 0, \\
\varepsilon\left\|D^{m} u\right\|^{2 q+2}-\varepsilon\left\|D^{m} u\right\|^{2}+q \varepsilon \geq 0 .
\end{gathered}
$$

By (2.22), we get

$$
\begin{aligned}
& \left(2 \lambda_{1}^{m}-2 \varepsilon^{2}-2 \varepsilon\right)\|v\|^{2}-\varepsilon\left\|D^{m} u\right\|^{2}+2 \varepsilon\left\|D^{m} u\right\|^{2 q+2}+2 C_{2} \varepsilon J(u)+q \varepsilon \\
= & \left(2 \lambda_{1}^{m}-2 \varepsilon^{2}-2 \varepsilon\right)\|v\|^{2}+\varepsilon(q+1)\left(\frac{1}{q+1}\left\|D^{m} u\right\|^{2 q+2}\right) \\
& +\left(\varepsilon\left\|D^{m} u\right\|^{2 q+2}-\varepsilon\left\|D^{m} u\right\|^{2}+q \varepsilon\right)+2 C_{2} \varepsilon J(u) \\
\geq & w\left(\|v\|^{2}+\frac{1}{q+1}\left\|D^{m} u\right\|^{2 q+2}\right)+2 C_{2} \varepsilon J(u) \\
\geq & w\left(\|v\|^{2}-\varepsilon\left\|D^{m} u\right\|^{2}+\frac{1}{q+1}\left\|D^{m} u\right\|^{2 q+2}\right)+2 C_{2} \varepsilon J(u),
\end{aligned}
$$

where $w=\min \left\{2 \lambda_{1}^{m}-2 \varepsilon^{2}-2 \varepsilon,(q+1) \varepsilon\right\}$. 
By (2.21) and substituting (2.23) into (2.20), we receive

$$
\begin{aligned}
& \frac{\mathrm{d}}{\mathrm{d} t}\left(\|v\|^{2}-\varepsilon\left\|D^{m} u\right\|^{2}+\frac{1}{q+1}\left\|D^{m} u\right\|^{2 q+2}+\frac{q}{q+1}+2 J(u)\right) \\
& +w\left(\|v\|^{2}-\varepsilon\left\|D^{m} u\right\|^{2}+\frac{1}{q+1}\left\|D^{m} u\right\|^{2 q+2}+\frac{q}{q+1}\right)+2 C_{2} \varepsilon J(u) \\
& \leq \frac{1}{\varepsilon^{2}}\|f\|^{2}+2 \varepsilon C\left(\gamma_{1}\right)+q \varepsilon+\frac{q w}{q+1} .
\end{aligned}
$$

Since $0<\varepsilon<\frac{\sqrt{\left(2+C_{2}\right)^{2}+16 \lambda_{1}^{m}}-2-C_{2}}{4}$ and $0<C_{2}<1$, we get

$$
w=\min \left\{2 \lambda_{1}^{m}-2 \varepsilon^{2}-2 \varepsilon,(q+1) \varepsilon\right\} \geq C_{2} \varepsilon .
$$

By (2.6) and (2.21), we have

$$
\begin{aligned}
& -\varepsilon\left\|D^{m} u\right\|^{2}+\frac{1}{q+1}\left\|D^{m} u\right\|^{2 q+2}+\frac{q}{q+1}+2 J(u)+2 C\left(\gamma_{2}\right) \\
& \geq(1-\varepsilon)\left\|D^{m} u\right\|^{2}+2 J(u)+2 C\left(\gamma_{2}\right) \geq 0,
\end{aligned}
$$

where $\gamma_{2}=\frac{1-\varepsilon}{2}>0$.

Combining with (2.25) and (2.26), formula (2.24) into

$$
\begin{aligned}
& \frac{\mathrm{d}}{\mathrm{d} t}\left(\|v\|^{2}-\varepsilon\left\|D^{m} u\right\|^{2}+\frac{1}{q+1}\left\|D^{m} u\right\|^{2 q+2}+\frac{q}{q+1}+2 J(u)+2 C\left(\gamma_{2}\right)\right) \\
& +C_{2} \varepsilon\left(\|v\|^{2}-\varepsilon\left\|D^{m} u\right\|^{2}+\frac{1}{q+1}\left\|D^{m} u\right\|^{2 q+2}+\frac{q}{q+1}+2 J(u)+2 C\left(\gamma_{2}\right)\right) \\
& \leq \frac{1}{\varepsilon^{2}}\|f\|^{2}+2 \varepsilon C\left(\gamma_{1}\right)+q \varepsilon+\frac{q w}{q+1}+2 \varepsilon C_{2} C\left(\gamma_{2}\right) .
\end{aligned}
$$

We set $y(t)=\|v\|^{2}-\varepsilon\left\|D^{m} u\right\|^{2}+\frac{1}{q+1}\left\|D^{m} u\right\|^{2 q+2}+\frac{q}{q+1}+2 J(u)+2 C\left(\gamma_{2}\right)$. Then, (2.27) is simplified as

$$
\frac{\mathrm{d}}{\mathrm{d} t} y(t)+C_{2} \varepsilon y(t) \leq \tilde{C},
$$

where $\tilde{C}=\frac{1}{\varepsilon^{2}}\|f\|^{2}+2 \varepsilon C\left(\gamma_{1}\right)+q \varepsilon+\frac{q w}{q+1}+2 \varepsilon C_{2} C\left(\gamma_{2}\right)$.

From conclusion (2.26), we know $y(t) \geq 0$. So, by Gronwall's inequality, we obtain

$$
y(t) \leq y(0) \mathrm{e}^{-C_{2} \varepsilon t}+\frac{\tilde{C}}{C_{2} \varepsilon},
$$

where $y(0)=\left\|u_{1}+\varepsilon u_{0}\right\|^{2}-\varepsilon\left\|D^{m} u_{0}\right\|^{2}+\frac{1}{q+1}\left\|D^{m} u_{0}\right\|^{2 q+2}+\frac{q}{q+1}+2 J\left(u_{0}\right)+\underset{q+1}{2 C\left(\gamma_{2}\right) \text {. }}$

By generalized Young's inequality, we have $\left\|D^{m} u\right\|^{2} \leq \frac{1}{2^{q+1}(q+1)}\left\|D^{m} u\right\|^{2 q+2}+2^{\frac{q+1}{q}} \frac{q}{q+1}$. Then, we get

$$
\frac{1}{(q+1)}\left\|D^{m} u\right\|^{2 q+2} \geq 2^{q+1}\left\|D^{m} u\right\|^{2}-2^{\frac{q^{2}+2 q+1}{q}} \frac{q}{q+1} .
$$


By (2.26) and (2.30), we have

$$
\begin{aligned}
y(t)= & \|v\|^{2}-\varepsilon\left\|D^{m} u\right\|^{2}+\frac{1}{q+1}\left\|D^{m} u\right\|^{2 q+2}+2 J(u)+\frac{q}{q+1}+2 C\left(\gamma_{2}\right) \\
\geq & \|v\|^{2}+(1-\varepsilon)\left\|D^{m} u\right\|^{2}+\left(2^{q+1}-1\right)\left\|D^{m} u\right\|^{2} \\
& -\frac{2^{q^{2}+2 q+1} q}{q+1}+\frac{q}{q+1}+2 J(u)+2 C\left(\gamma_{2}\right) \\
\geq & \|v\|^{2}+\left(2^{q+1}-1\right)\left\|D^{m} u\right\|^{2}+\left(1-2^{q^{2}+2 q+1}\right) \frac{q}{q+1} \\
\geq & \min \left\{1,2^{q+1}-1\right\}\left(\|v\|^{2}+\left\|D^{m} u\right\|^{2}\right)+\left(1-2^{q^{2}+2 q+1}\right) \frac{q}{q+1} \\
= & \left(\|v\|^{2}+\left\|D^{m} u\right\|^{2}\right)+\left(1-2^{q^{2}+2 q+1}\right) \frac{q}{q+1} .
\end{aligned}
$$

Combining with (2.29) and (2.31),we obtain

$$
\left\|D^{m} u\right\|^{2}+\|v\|^{2} \leq y(0) \mathrm{e}^{-C_{2} \varepsilon t}+\frac{\tilde{C}}{C_{2} \varepsilon}+\left(2^{q^{2}+2 q+1}-1\right) \frac{q}{q+1},
$$

Then,

$$
\varlimsup_{t \rightarrow \infty}\|(u, v)\|_{H_{0}^{m}(\Omega) \times L^{2}(\Omega)}^{2}=\left\|D^{m} u\right\|^{2}+\|v\|^{2} \leq \frac{\tilde{C}}{C_{2} \varepsilon}+\left(2^{q^{2}+2 q+1}-1\right) \frac{q}{q+1} .
$$

So, there exist $E_{0}$ and $t_{0}=t_{0}(\Omega)>0$, such that

$$
\|(u, v)\|_{H_{0}^{m}(\Omega) \times L^{2}(\Omega)}^{2}=\left\|D^{m} u\right\|^{2}+\|v\|^{2} \leq E_{0}, \quad\left(t>t_{0}\right) .
$$

Lemma 2. In addition to the assumptions of Lemma 1, $\left(\mathrm{H}_{1}\right)-\left(\mathrm{H}_{4}\right)$ hold. If $\left(\mathrm{H}_{5}\right)$ : $f(x) \in H_{0}^{m}(\Omega)$, and $\left(u_{0}, u_{1}\right) \in H^{2 m}(\Omega) \times H_{0}^{m}(\Omega)$. Then the solution $(u, v)$ of the problems $(1.1)-(1.3)$ satisfies $(u, v) \in L^{\infty}\left((0,+\infty) ; H^{2 m}(\Omega) \times H_{0}^{m}(\Omega)\right)$, and

$$
\left\|D^{2 m} u\right\|^{2}+\left\|D^{m} v\right\|^{2} \leq \frac{z(0)}{T} \mathrm{e}^{-\alpha_{1} t}+\frac{\frac{1}{\varepsilon^{2}}\left\|D^{m} f\right\|^{2}+C_{3}}{\alpha_{1} T} .
$$

where $(-\Delta)^{m} v=(-\Delta)^{m} u_{t}+\varepsilon(-\Delta)^{m} u, \lambda_{1}$ is the first eigenvalue of $-\Delta$ in $H_{0}^{1}(\Omega)$, and $z(0)=\left\|D^{m} u_{1}+\varepsilon D^{m} u_{0}\right\|^{2}+\left(\left\|D^{m} u_{0}\right\|^{2 q}-\varepsilon\right)\left\|D^{2 m} u_{0}\right\|^{2}, \quad \alpha_{1}=\min \left\{\lambda_{1}^{m}-2 \varepsilon-2 \varepsilon^{2}, M\right\}$, $T=\min \left\{1, \inf _{t \geq 0}\left\|D^{m} u\right\|^{2 q}-\varepsilon\right\}$. Thus, there exists $E_{1}$ and $t_{1}=t_{1}(\Omega)>0$, such that

$$
\|(u, v)\|_{H^{2 m}(\Omega) \times H_{0}^{m}(\Omega)}^{2}=\left\|D^{2 m} u\right\|^{2}+\left\|D^{m} v\right\|^{2} \leq E_{1}, \quad\left(t>t_{1}\right) .
$$

Proof. Taking $L^{2}$-inner product by $(-\Delta)^{m} v=(-\Delta)^{m} u_{t}+\varepsilon(-\Delta)^{m} u$ in (1.1), we have

$$
\left(u_{t t}+(-\Delta)^{m} u_{t}+\left\|D^{m} u\right\|^{2 q}(-\Delta)^{m} u+g(u),(-\Delta)^{m} v\right)=\left(f(x),(-\Delta)^{m} v\right) \text {. }
$$

After a computation in (2.37) one by one, as follow

$$
\begin{aligned}
\left(u_{t t},(-\Delta)^{m} v\right) & =\frac{1}{2} \frac{\mathrm{d}}{\mathrm{d} t}\left\|D^{m} v\right\|^{2}-\varepsilon\left\|D^{m} v\right\|^{2}+\varepsilon^{2}\left(D^{m} u, D^{m} v\right) \\
& \geq \frac{1}{2} \frac{\mathrm{d}}{\mathrm{d} t}\left\|D^{m} v\right\|^{2}-\varepsilon\left\|D^{m} v\right\|^{2}-\frac{\varepsilon^{2}}{2 \lambda_{1}^{m}}\left\|D^{2 m} u\right\|^{2}-\frac{\varepsilon^{2}}{2}\left\|D^{m} v\right\|^{2},
\end{aligned}
$$




$$
\begin{gathered}
\left((-\Delta)^{m} u_{t},(-\Delta)^{m} v\right)=\left\|D^{2 m} v\right\|^{2}-\frac{\varepsilon}{2} \frac{\mathrm{d}}{\mathrm{d} t}\left\|D^{2 m} u\right\|^{2}-\varepsilon^{2}\left\|D^{2 m} u\right\|^{2}, \\
\left(\left\|D^{m} u\right\|^{2 q}(-\Delta)^{m} u,(-\Delta)^{m} v\right) \\
=\frac{1}{2} \frac{\mathrm{d}}{\mathrm{d} t}\left(\left\|D^{m} u\right\|^{2 q}\left\|D^{2 m} u\right\|^{2}\right)-\frac{\left\|D^{2 m} u\right\|^{2}}{2} \frac{\mathrm{d}}{\mathrm{d} t}\left\|D^{m} u\right\|^{2 q}+\varepsilon\left\|D^{m} u\right\|^{2 q}\left\|D^{2 m} u\right\|^{2} .
\end{gathered}
$$

By Young's inequality, we get

$$
\left(g(u),(-\Delta)^{m} v\right) \geq-\|g(u)\|\left\|D^{2 m} v\right\| \geq-\frac{\|g(u)\|^{2}}{2}-\frac{\left\|D^{2 m} v\right\|^{2}}{2} .
$$

Next to estimate $\|g(u)\|^{2}$ in (2.41). By $\left(\mathrm{H}_{4}\right):|g(s)| \leq C_{1}\left(1+|s|^{p}\right)$ and Young's inequality, we have

$$
\begin{aligned}
\|g(u)\|^{2} & \leq \int_{\Omega} C_{1}^{2}\left(1+|u|^{p}\right)^{2} \mathrm{~d} x \\
& \leq \int_{\Omega}\left(C_{1}^{2}+2 C_{1}^{2}|u|^{p}+C_{1}^{2}|u|^{2 p}\right) \mathrm{d} x \\
& \leq \int_{\Omega}\left(2 C_{1}^{2}+2 C_{1}^{2}|u|^{2 p}\right) \mathrm{d} x \\
& \leq 2 C_{1}^{2}|\Omega|+2 C_{1}^{2}\|u\|_{L^{2 p}(\Omega)}^{2 p} .
\end{aligned}
$$

By $1 \leq p \leq \frac{n}{n-2 m}$ and Embeding Theorem, then $H_{0}^{m}(\Omega) \rightarrow L^{2 p}(\Omega)$. So there exists $K>0$, such that $\|u\|_{L^{2 p}(\Omega)} \leq K\left\|D^{m} u\right\| \cdot\left\|D^{m} u\right\|$ bounded by lemma 1 . Then, (2.42) turns into

$$
\|g(u)\|^{2} \leq C_{3}\left(p, C_{1}, K,|\Omega|\right) .
$$

Collecting with (2.43), from (2.41) we have

$$
\left(g(u),(-\Delta)^{m} v\right) \geq-\frac{C_{3}}{2}-\frac{\left\|D^{2 m} v\right\|^{2}}{2} .
$$

By $f(x) \in H_{0}^{m}(\Omega)$ and Young's inequality, we obtain

$$
\left(f(x),(-\Delta)^{m} v\right)=\left(D^{m} f(x), D^{m} v\right) \leq \frac{1}{2 \varepsilon^{2}}\left\|D^{m} f\right\|^{2}+\frac{\varepsilon^{2}}{2}\left\|D^{m} v\right\|^{2} .
$$

Integrating (2.38) - (2.40), (2.44) - (2.45), from (2.37) entails

$$
\begin{aligned}
& \frac{\mathrm{d}}{\mathrm{d} t}\left[\left\|D^{m} v\right\|^{2}+\left(\left\|D^{m} u\right\|^{2 q}-\varepsilon\right)\left\|D^{2 m} u\right\|^{2}\right]+\left\|D^{2 m} v\right\|^{2}-\left(2 \varepsilon+2 \varepsilon^{2}\right)\left\|D^{m} v\right\|^{2} \\
& +\left(-\frac{\mathrm{d}}{\mathrm{d} t}\left\|D^{m} u\right\|^{2 q}+2 \varepsilon\left\|D^{m} u\right\|^{2 q}-2 \varepsilon^{2}-\frac{\varepsilon^{2}}{\lambda_{1}^{m}}\right)\left\|D^{2 m} u\right\|^{2} \leq \frac{1}{\varepsilon^{2}}\left\|D^{m} f\right\|^{2}+C_{3}
\end{aligned}
$$

By Poincaré inequality, such that $\lambda_{1}^{m}\left\|D^{m} v\right\|^{2} \leq\left\|D^{2 m} v\right\|^{2}$. So, (2.46) turns into

$$
\begin{aligned}
& \frac{\mathrm{d}}{\mathrm{d} t}\left[\left\|D^{m} v\right\|^{2}+\left(\left\|D^{m} u\right\|^{2 q}-\varepsilon\right)\left\|D^{2 m} u\right\|^{2}\right]+\left(\lambda_{1}^{m}-2 \varepsilon-2 \varepsilon^{2}\right)\left\|D^{m} v\right\|^{2} \\
& +\left(-\frac{\mathrm{d}}{\mathrm{d} t}\left\|D^{m} u\right\|^{2 q}+2 \varepsilon\left\|D^{m} u\right\|^{2 q}-2 \varepsilon^{2}-\frac{\varepsilon^{2}}{\lambda_{1}^{m}}\right)\left\|D^{2 m} u\right\|^{2} \leq \frac{1}{\varepsilon^{2}}\left\|D^{m} f\right\|^{2}+C_{3}
\end{aligned}
$$


First, we take proper $\varepsilon$, such that $\lambda_{1}^{m}-2 \varepsilon-2 \varepsilon^{2}>0$ and $\left\|D^{m} u\right\|^{2 q}-\varepsilon>0$ by Lamma 1 . Then, we assume that there exists $M>0$, such that $M-2 \varepsilon>0$ and $0<M\left(\left\|D^{m} u\right\|^{2 q}-\varepsilon\right) \leq-\frac{\mathrm{d}}{\mathrm{d} t}\left\|D^{m} u\right\|^{2 q}+2 \varepsilon\left\|D^{m} u\right\|^{2 q}-2 \varepsilon^{2}-\frac{\varepsilon^{2}}{\lambda_{1}^{m}}$. Then, formula is simplified to

$$
(M-2 \varepsilon)\left\|D^{m} u\right\|^{2 q}+\frac{\mathrm{d}}{\mathrm{d} t}\left\|D^{m} u\right\|^{2 q} \leq M \varepsilon-2 \varepsilon^{2}-\frac{\varepsilon^{2}}{\lambda_{1}^{m}} .
$$

By Gronwall's inequality, we get

$$
\varepsilon<\left\|D^{m} u\right\|^{2 q} \leq\left\|D^{m} u_{0}\right\|^{2 q} \mathrm{e}^{(2 \varepsilon-M) t}+\frac{M \varepsilon-2 \varepsilon^{2}-\frac{\varepsilon^{2}}{\lambda_{1}^{m}}}{M-2 \varepsilon} .
$$

On account of Lemma 1, we know $\left\|D^{m} u\right\|^{2 q}$ is bounded. So the hypothesis is true. Namely, we prove that there are $M>0$, makes

$$
0<M\left(\left\|D^{m} u\right\|^{2 q}-\varepsilon\right) \leq-\frac{\mathrm{d}}{\mathrm{d} t}\left\|D^{m} u\right\|^{2 q}+2 \varepsilon\left\|D^{m} u\right\|^{2 q}-2 \varepsilon^{2}-\frac{\varepsilon^{2}}{\lambda_{1}^{m}} .
$$

Substituting (2.50) into (2.47), we receive

$$
\begin{aligned}
& \frac{\mathrm{d}}{\mathrm{d} t}\left[\left\|D^{m} v\right\|^{2}+\left(\left\|D^{m} u\right\|^{2 q}-\varepsilon\right)\left\|D^{2 m} u\right\|^{2}\right]+\left(\lambda_{1}^{m}-2 \varepsilon-2 \varepsilon^{2}\right)\left\|D^{m} v\right\|^{2} \\
& +M\left(\left\|D^{m} u\right\|^{2 q}-\varepsilon\right)\left\|D^{2 m} u\right\|^{2} \leq \frac{1}{\varepsilon^{2}}\left\|D^{m} f\right\|^{2}+C_{3} .
\end{aligned}
$$

Taking $\alpha_{1}=\min \left\{\lambda_{1}^{m}-2 \varepsilon-2 \varepsilon^{2}, M\right\}$, then

$$
\frac{\mathrm{d}}{\mathrm{d} t} z(t)+\alpha_{1} z(t) \leq \frac{1}{\varepsilon^{2}}\left\|D^{m} f\right\|^{2}+C_{3},
$$

where $z(t)=\left\|D^{m} v\right\|^{2}+\left(\left\|D^{m} u\right\|^{2 q}-\varepsilon\right)\left\|D^{2 m} u\right\|^{2}$. By Gronwall's inequality, we have

$$
z(t) \leq z(0) \mathrm{e}^{-\alpha_{1} t}+\frac{\frac{1}{\varepsilon^{2}}\left\|D^{m} f\right\|^{2}+C_{3}}{\alpha_{1}},
$$

where $z(0)=\left\|D^{m} u_{1}+\varepsilon D^{m} u_{0}\right\|^{2}+\left(\left\|D^{m} u_{0}\right\|^{2 q}-\varepsilon\right)\left\|D^{2 m} u_{0}\right\|^{2}$.

Let $T=\min \left\{1, \inf _{t \geq 0}\left\|D^{m} u\right\|^{2 q}-\varepsilon\right\}$, so we get

$$
\left\|D^{m} v\right\|^{2}+\left\|D^{2 m} u\right\|^{2} \leq \frac{z(0)}{T} \mathrm{e}^{-\alpha_{1} t}+\frac{\frac{1}{\varepsilon^{2}}\left\|D^{m} f\right\|^{2}+C_{3}}{\alpha_{1} T},
$$

Then

$$
\varlimsup_{t \rightarrow \infty}\|(u, v)\|_{H^{2 m}(\Omega) \times H_{0}^{m}(\Omega)}^{2}=\left\|D^{2 m} u\right\|^{2}+\left\|D^{m} v\right\|^{2} \leq \frac{\frac{1}{\varepsilon^{2}}\left\|D^{m} f\right\|^{2}+C_{3}}{\alpha_{1} T} .
$$

So, there exists $E_{1}$ and $t_{1}=t_{1}(\Omega)>0$, such that

$$
\|(u, v)\|_{H^{2 m}(\Omega) \times H_{0}^{m}(\Omega)}^{2}=\left\|D^{2 m} u\right\|^{2}+\left\|D^{m} v\right\|^{2} \leq E_{1}, \quad\left(t>t_{1}\right) .
$$




\section{Global Attractor}

\subsection{The Existence and Uniqueness of Solution}

Theorem 3.1. Assume $\left(\mathrm{H}_{1}\right)-\left(\mathrm{H}_{4}\right)$ hold, and $\left(u_{0}, u_{1}\right) \in H^{2 m}(\Omega) \times H_{0}^{m}(\Omega)$, $f(x) \in H_{0}^{m}(\Omega), \quad v=u_{t}+\varepsilon u$. So Equation (1.1) exists a unique smooth solution

$$
(u(x, t), v(x, t)) \in L^{\infty}\left((0,+\infty) ; H^{2 m}(\Omega) \times H_{0}^{m}(\Omega)\right) .
$$

Proof. By the Galerkin method, Lemma 1 and Lemma 2, we can easily obtain the existence of Solutions. Next, we prove the uniqueness of Solutions in detail.

Assume $u, v$ are two solutions of the problems (1.1) - (1.3), let $w=u-v$, then $w(x, 0)=w_{0}(x)=0, w_{t}(x, 0)=w_{1}(x)=0$ and the two equations subtract and obtain

$$
w_{t t}+(-\Delta)^{m} w_{t}+\left\|D^{m} u\right\|^{2 q}(-\Delta)^{m} u-\left\|D^{m} v\right\|^{2 q}(-\Delta)^{m} v+g(u)-g(v)=0 .
$$

By multiplying (3.2) by $w_{t}$, we get

$$
\begin{gathered}
\left(w_{t t}+(-\Delta)^{m} w_{t}+\left\|D^{m} u\right\|^{2 q}(-\Delta)^{m} u-\left\|D^{m} v\right\|^{2 q}(-\Delta)^{m} v+g(u)-g(v), w_{t}\right)=0, \\
\left(w_{t t}, w_{t}\right)=\frac{1}{2} \frac{\mathrm{d}}{\mathrm{d} t}\left\|w_{t}\right\|^{2}, \\
\left((-\Delta)^{m} w_{t}, w_{t}\right)=\left\|D^{m} w_{t}\right\|^{2}, \\
\left(\left\|D^{m} u\right\|^{2 q}(-\Delta)^{m} u-\left\|D^{m} v\right\|^{2 q}(-\Delta)^{m} v, w_{t}\right) \\
=\left(\left\|D^{m} u\right\|^{2 q}(-\Delta)^{m} w, w_{t}\right)+\left(\left\|D^{m} u\right\|^{2 q}-\left\|D^{m} v\right\|^{2 q}\right)\left((-\Delta)^{m} v, w_{t}\right) \\
=\frac{1}{2} \frac{\mathrm{d}}{\mathrm{d} t}\left\|D^{m} u\right\|^{2 q}\left\|D^{m} w\right\|^{2}-q\left\|D^{m} u\right\|^{2 q-1}\left\|D^{m} u_{t}\right\|\left\|D^{m} w\right\|^{2} \\
+\left(\left\|D^{m} u\right\|^{2 q}-\left\|D^{m} v\right\|^{2 q}\right)\left((-\Delta)^{m} v, w_{t}\right) .
\end{gathered}
$$

Exploiting (3.4) - (3.6), we receive

$$
\begin{aligned}
& \frac{\mathrm{d}}{\mathrm{d} t}\left(\left\|w_{t}\right\|^{2}+\left\|D^{m} u\right\|^{2 q}\left\|D^{m} w\right\|^{2}\right)+2\left\|D^{m} w_{t}\right\|^{2} \\
& =2 q\left\|D^{m} u\right\|^{2 q-1}\left\|D^{m} u_{t}\right\|\left\|D^{m} w\right\|^{2}-2\left(\left\|D^{m} u\right\|^{2 q}-\left\|D^{m} v\right\|^{2 q}\right)\left((-\Delta)^{m} v, w_{t}\right) \\
& \quad-2\left(g(u)-g(v), w_{t}\right) .
\end{aligned}
$$

In (3.7), according to Lemma 1 and Lemma 2, such that

$$
\begin{aligned}
& 2 q\left\|D^{m} u\right\|^{2 q-1}\left\|D^{m} w\right\|^{2}-2\left(\left\|D^{m} u\right\|^{2 q}-\left\|D^{m} v\right\|^{2 q}\right)\left((-\Delta)^{m} v, w_{t}\right) \\
& \leq 2 q\left\|D^{m} u\right\|^{2 q-1}\left\|D^{m} w\right\|^{2}+4 q\left\|D^{m} u\right\|+\left.\theta\left(\left\|D^{m} v\right\|-\left\|D^{m} u\right\|\right)\right|^{2 q-1}\left\|D^{m} w\right\|\left\|\Delta^{m} v\right\|\left\|w_{t}\right\| \\
& \leq C_{4}(q)\left\|D^{m} w\right\|^{2}+C_{5}(q, \theta)\left\|D^{m} w\right\|\left\|w_{t}\right\| \\
& \leq\left(C_{4}(q)+\frac{C_{5}(q, \theta)}{2}\right)\left\|D^{m} w\right\|^{2}+\frac{C_{5}(q, \theta)}{2}\left\|w_{t}\right\|^{2},
\end{aligned}
$$

where $0<\theta<1, C_{4}(q)>0$ and $C_{5}(q, \theta)>0$ are constants. 
By $\left(\mathrm{H}_{4}\right)$, we obtain

$$
\begin{aligned}
& -2\left(g(u)-g(v), w_{t}\right) \\
& =-2\left(g^{\prime}(\theta u+(1-\theta) v) w, w_{t}\right) \\
& \leq 2\left\|g^{\prime}(\theta u+(1-\theta) v) w\right\|\left\|w_{t}\right\| \\
& \leq 2\left(\int_{\Omega} C_{1}^{2}\left(1+|\theta u+(1-\theta) v|^{p-1} d x\right)^{\frac{1}{2}}\|w\|\left\|w_{t}\right\|\right. \\
& \leq 2 C_{1}\left(1+\left\|\left(|\theta u+(1-\theta) v|^{p-1}\right)\right\|\right)\|w\|\left\|w_{t}\right\| \\
& \leq 2 C_{1} C_{6}\left(\theta, p, \lambda_{1}, m\right)\left\|D^{m} w\right\|\left\|w_{t}\right\| \\
& \leq C_{1} C_{6}\left(\theta, p, \lambda_{1}, m\right)\left\|w_{t}\right\|^{2}+C_{1} C_{6}\left(\theta, p, \lambda_{1}, m\right)\left\|D^{m} w\right\|^{2},
\end{aligned}
$$

where $C_{6}=C_{6}\left(\theta, p, \lambda_{1}, m\right)>0$ is constant.

From the above, we have

$$
\frac{\mathrm{d}}{\mathrm{d} t}\left(\left\|w_{t}\right\|^{2}+\left\|D^{m} u\right\|^{2 q}\left\|D^{m} w\right\|^{2}\right) \leq\left(C_{4}(q)+\frac{C_{5}(\theta, q)}{2}+C_{1} C_{6}\right)\left(\left\|w_{t}\right\|^{2}+\left\|D^{m} w\right\|^{2}\right) .
$$

For (3.10), because $\left\|D^{m} u\right\|^{2 q}$ is bounded. Then, there exists $\varepsilon>0$, such that $\left\|D^{m} u\right\|^{2 q} \geq \varepsilon$. So, we have

$$
\begin{aligned}
& \frac{\mathrm{d}}{\mathrm{d} t}\left(\left\|w_{t}\right\|^{2}+\left\|D^{m} u\right\|^{2 q}\left\|D^{m} w\right\|^{2}\right) \\
& \left.\leq\left(C_{4}(q)+\frac{C_{5}(\theta, q)}{2}+C_{1} C_{6}\right)\right)\left\|w_{t}\right\|^{2} \\
& \quad+\left(\frac{C_{4}(q)}{\varepsilon}+\frac{C_{5}(\theta, q)}{2 \varepsilon}+\frac{C_{1} C_{6}}{\varepsilon}\right)\left\|D^{m} u\right\|^{2 q}\left\|D^{m} w\right\|^{2} \\
& \leq C_{7}\left(\left\|w_{t}\right\|^{2}+\left\|D^{m} u\right\|^{2 q}\left\|D^{m} w\right\|^{2}\right),
\end{aligned}
$$

where $C_{7}=\min \left\{C_{4}(q)+\frac{C_{5}(\theta, q)}{2}+C_{1} C_{6}, \frac{C_{4}(q)}{\varepsilon}+\frac{C_{5}(\theta, q)}{2 \varepsilon}+\frac{C_{1} C_{6}}{\varepsilon}\right\}$. By using Gronwall's inequality for (3.11), we obtain

$$
0 \leq\left\|w_{t}\right\|^{2}+\left\|D^{m} u\right\|^{2 a}\left\|D^{m} w\right\|^{2} \leq\left(\left\|w_{t}(0)\right\|^{2}+\left\|D^{m} u(0)\right\|^{2 q}\left\|D^{m} w(0)\right\|^{2}\right) \mathrm{e}^{C_{7} t}=0 .
$$

Hence, we can get $\left\|w_{t}\right\|^{2}+\left\|D^{m} u\right\|^{2 q}\left\|D^{m} w\right\|^{2}=0$. That shows that

$$
\left\|w_{t}\right\|^{2}=0, \quad\left\|D^{m} u\right\|^{2 q}\left\|D^{m} w\right\|^{2}=0 .
$$

That is

$$
w(x, t)=0 .
$$

Therefore

$$
u=v .
$$

So we get the uniqueness of the solution. 


\subsection{Global Attractor}

Theorem 3.2. [10] Let $E$ be a Banach space, and $\{S(t)\}(t \geq 0)$ are the semigroup operator on $E$. $S(t): E \rightarrow E, S(t+\tau)=S(t) S(\tau)(\forall t, \tau \geq 0), S(0)=I$, where $I$ is a unit operator.Set $S(t)$ satisfy the follow conditions:

1) $S(t)$ is uniformly bounded, namely $\forall R>0,\|u\|_{E} \leq R$, it exists a constant $C(R)$, so that

$$
\|S(t) u\|_{E} \leq C(R) \quad(t \in[0,+\infty)) ;
$$

2) It exists a bounded absorbing set $B_{0} \subset E$, namely, $\forall B \subset E$, it exists a constant $t_{0}$, so that

$$
S(t) B \subset B_{0} \quad\left(t \geq t_{0}\right) ;
$$

where $B_{0}$ and $B$ are bounded sets.

3) When $t>0, S(t)$ is a completely continuous operator. Therefore, the semigroup operator $\mathrm{S}(\mathrm{t})$ exists a compact global attractor $\mathcal{A}$.

Theorem 3.3. Under the assume of Lemma 1, Lemma 2 and Theorem 3.1, equations have global attractor

$$
\mathcal{A}=\omega\left(B_{0}\right)=\bigcap_{\tau \geq 0} \overline{\bigcup_{t \geq \tau} S(t) B_{0}},
$$

where $B_{0}=\left\{(u, v) \in H^{2 m}(\Omega) \times H_{0}^{m}(\Omega):\|(u, v)\|_{H^{2 m} \times H_{0}^{m}}^{2}=\|u\|_{H^{2 m}}^{2}+\|v\|_{H_{0}^{m}}^{2} \leq R_{0}+R_{1}\right\}, \quad B_{0}$ is the bounded absorbing set of $H^{2 m} \times H_{0}^{m}$ and satisfies

1) $S(t) \mathcal{A}=\mathcal{A}, t>0$;

2) $\lim _{t \rightarrow \infty} \operatorname{dist}(S(t) B, \mathcal{A})=0$, here $B \subset H^{2 m} \times H_{0}^{m}$ and it is a bounded set,

$$
\operatorname{dist}(S(t) B, \mathcal{A})=\sup _{x \in B}\left(\inf _{y \in \mathcal{A}}\|S(t) x-y\|_{H^{2 m} \times H_{0}^{m}}\right) \rightarrow 0, t \rightarrow \infty .
$$

Proof. Under the conditions of Theorem 3.1, it exists the solution semigroup $\mathrm{S}(\mathrm{t})$, $S(t): H^{2 m} \times H_{0}^{m} \rightarrow H^{2 m} \times H_{0}^{m}$, here $E=H^{2 m}(\Omega) \times H_{0}^{m}(\Omega)$.

(1) From Lemma 1 to Lemma 2, we can get that $\forall B \subset H^{2 m}(\Omega) \times H_{0}^{m}(\Omega)$ is a bounded set that includes in the ball $\left\{\|(u, v)\|_{H^{2 m} \times H_{0}^{m}} \leq R\right\}$,

$$
\begin{aligned}
\left\|S(t)\left(u_{0}, v_{0}\right)\right\|_{H^{2 m} \times H_{0}^{m}}^{2} & =\|u\|_{H^{2 m}}^{2}+\|v\|_{H_{0}^{m}}^{2} \leq\left\|u_{0}\right\|_{H^{2 m}}^{2}+\left\|v_{0}\right\|_{H_{0}^{m}}^{2}+C \\
& \leq R^{2}+C,\left(t \geq 0,\left(u_{0}, v_{0}\right) \in B\right) .
\end{aligned}
$$

This shows that $S(t)(t \geq 0)$ is uniformly bounded in $H^{2 m}(\Omega) \times H_{0}^{m}(\Omega)$.

(2) Furthermore, for any $\left(u_{0}, v_{0}\right) \in H^{2 m}(\Omega) \times H_{0}^{m}(\Omega)$, when $t \geq \max \left\{t_{0}, t_{1}\right\}$, we have

$$
\left\|S(t)\left(u_{0}, v_{0}\right)\right\|_{H^{2 m} \times H_{0}^{m}}^{2}=\|u\|_{H^{2 m}}^{2}+\|v\|_{H_{0}^{m}}^{2} \leq R_{0}+R_{1} .
$$

So we get $B_{0}$ is the bounded absorbing set.

(3) Since $E_{1}:=H^{2 m}(\Omega) \times H_{0}^{m}(\Omega) \rightarrow E_{0}:=H^{2 m}(\Omega) \times L^{2}(\Omega)$ is compact embedded, which means that the bounded set in $E_{1}$ is the compact set in $E_{0}$, so the semigroup operator $\mathrm{S}(\mathrm{t})$ exists a compact global attractor $\mathcal{A}$. 


\section{The Estimates of the Upper Bounds of Hausdorff and Fractal Dimensions for the Global Attractor}

We rewrite the problems (1.1) - (1.3):

$$
\begin{gathered}
u_{t t}+A^{m} u_{t}+\left\|A^{\frac{m}{2}} u\right\|^{2 q} A^{m} u+g(u)=f(x) \quad \text { in } \Omega \times R^{+}, \\
u(x, 0)=u_{0}(x) ; u_{t}(x, 0)=u_{1}(x), \quad x \in \Omega, \\
\left.u(x, t)\right|_{\partial \Omega}=0, \frac{\partial^{i} u}{\partial v^{i}}=0(i=1, \cdots, m-1), \quad \text { in } \partial \Omega \times R^{+} .
\end{gathered}
$$

Let $A u=-\Delta u$, where $\Omega$ is a bounded domain in $\mathbb{R}^{N}$ with smooth boundary $\partial \Omega, q$ is positive constant, and $m$ is positive integer. The linearized equations of the above equations as follows:

$$
\begin{gathered}
U_{t}+A U=F U, \\
U_{0}=\xi, U_{t}(0)=\zeta .
\end{gathered}
$$

Let $U_{0} \in H_{0}^{m}(\Omega), U(t)$ is the solution of problems (4.4) - (4.5). We can prove that the problems (4.4) - (4.5) have a unique solution $U \in L^{\infty}\left(0, T, H_{0}^{m}(\Omega)\right), U_{t} \in L^{\infty}\left(0, T, L^{2}(\Omega)\right)$. The equation (4.4) is the linearized equation by the Equation (4.17). Define the mapping $L s(t)_{u_{0}}: L s(t)_{u_{0}} \zeta=U(t)$, here $u(t)=s(t) u_{0}$, let $\varphi_{0}=\left(u_{0}, u_{1}\right)$,

$\overline{\varphi_{0}}=\varphi_{0}+\{\xi, \zeta\}=\left\{u_{0}+\xi, u_{1}+\zeta\right\}$, let $\left\|\varphi_{0}\right\|_{E_{0}} \leq R_{1},\left\|\overline{\varphi_{0}}\right\|_{E_{0}} \leq R_{2}, E_{0}=H_{0}^{m}(\Omega) \times \mathfrak{七}^{2}(\Omega)$, $S(t) \varphi_{0}=\varphi(t)=\left\{u(t), u_{t}(t)\right\}, S(t) \overline{\varphi_{0}}=\left\{\varphi(t), \overline{\varphi_{t}}(t)\right\}$.

Lemma 4.1 [6] Assume $H$ is a Hilbert space, $E_{0}$ is a compact set of $H$. $S(t): E_{0} \rightarrow H$ is a continuous mapping, satisfy the follow conditions.

1) $S(t) E_{0}=E_{0}, t>0$;

2) If $S(t)$ is Fréchet differentiable, it exists is a bounded linear differential operator $L\left(t, \varphi_{0}\right) \in C\left(R^{+} ; L\left(E_{0}, E_{0}\right)\right), \forall t>0$, that is

$$
\frac{\left\|S(t) \overline{\varphi_{0}}-S(t) \varphi_{0}-L\left(t, \varphi_{0}\right)(u, v)\right\|_{E_{0}}^{2}}{\|\{\xi, \zeta\}\|_{E_{0}}^{2}} \rightarrow 0,\{\xi, \zeta\} \rightarrow 0 .
$$

The proof of lemma 4.1 see ref. [6] is omitted here. According to Lemma 4.1, we can get the following theorem :

Theorem 4.1. [6] [7] Let $\mathcal{A}$ is the global attractor that we obtain in section 3.In that case, $\mathcal{A}$ has finite Hausdorff dimensions and Fractal dimensions in $H^{2 m}(\Omega) \times H_{0}^{m}(\Omega)$, that is $d_{H}(\mathcal{A}) \leq \frac{n}{5}, d_{F}(\mathcal{A}) \leq \frac{6 n}{5}$.

Proof. Firstly, we rewrite the equations (4.1), (4.2) into the first order abstract evolution equations in $E_{0}$.

Let $\Psi=R_{\varepsilon} \varphi=\left\{u, u_{t}+\varepsilon u\right\}$, let $R_{\varepsilon}:\left\{u, u_{t}\right\} \rightarrow\left\{u, u_{t}+\varepsilon u\right\}$, is an isomorphic mapping. So let $\mathcal{A}$ is the global attractor of $\{S(t)\}$, then $R_{\varepsilon} \mathcal{A}$ is also the global attractor of $\left\{S_{\varepsilon}(t)\right\}$, and they have the same dimensions. Then $\Psi$ satisfies as follows: 


$$
\begin{aligned}
& \Psi_{t}+\Lambda_{\varepsilon} \Psi+\bar{g}(\Psi)=\bar{f}, \\
& \Psi(0)=\left\{u_{0}, u_{1}+\varepsilon u_{0}\right\}^{\mathrm{T}},
\end{aligned}
$$

where $\Psi=\left\{u, u_{t}+\varepsilon u\right\}^{\mathrm{T}}, \bar{g}(\Psi)=\{0, g(u)\}^{\mathrm{T}}, \bar{f}=\{0, f(x)\}^{\mathrm{T}}$,

$$
\begin{gathered}
\Lambda_{\varepsilon}=\left(\| A^{\frac{m}{2} u \|^{2 q}} \begin{array}{cc}
\varepsilon I & -I \\
A^{m}-\varepsilon A^{m}+\varepsilon^{2} I & A^{m}-\varepsilon I
\end{array}\right), \\
\Psi_{t}:=F(\Psi)=\bar{f}-\Lambda_{\varepsilon} \Psi-\bar{g}(\Psi), \\
P_{t}=F_{t}(\Psi), \\
P_{t}+\Lambda_{\varepsilon} P+\bar{g}_{t}(\Psi) P=0
\end{gathered}
$$

where $P=\left\{U, U_{t}+\varepsilon U\right\}^{\mathrm{T}}, \bar{g}_{t}(\Psi) P=\left\{0, g_{t}(u) U\right\}^{\mathrm{T}}$. The initial condition (4.5) can be written in the following form:

$$
P(0)=\omega, \omega=\{\xi, \zeta\} \in E_{0} .
$$

We take $n \in N$, then consider the corresponding $n$ solutions: $\left(P=P_{1}, P_{2}, \cdots, P_{n} ; P_{j} \in E_{0}\right)$ of the initial values: $\left(\omega=\omega_{1}, \omega_{2}, \cdots, \omega_{n} ; \omega_{j} \in E_{0}\right)$ in the Equations (4.10) - (4.11). So there is $\left|P_{1}(t) \wedge P_{2}(t) \wedge \cdots \wedge P_{n}(t)\right|_{\wedge_{E_{0}}^{n}}=\left|\omega_{1} \wedge \omega_{2} \wedge \cdots \wedge \omega_{n}\right|_{\wedge_{E_{0}}^{n}} \cdot \mathrm{e}^{\int_{0}^{t} T r F_{t}\left(S_{\varepsilon}(\tau) \Psi_{0}\right) \cdot Q_{n}(\tau) \mathrm{d} \tau}$. from $\psi(\tau)=S_{\varepsilon}(\tau) \Psi_{0}$, we get $S_{\varepsilon}(\tau):\left\{u_{0}, v_{1}=u_{1}+\varepsilon u_{0}\right\} \rightarrow\left\{u(\tau), v(\tau)=u_{t}(\tau)+\varepsilon u(\tau)\right\}$, $\psi(\tau)=\left\{u(\tau), v_{t}(\tau)=u_{t}(\tau)+\varepsilon u(\tau)\right\}$, here $u$ is the solution of problems (4.1)-(4.3); $\wedge$ represents the outer product, $\operatorname{Tr}$ reprsents the trace, $Q_{n}(\tau)=Q_{n}\left(\tau, \Psi_{0} ; \omega_{1}, \omega_{2}, \cdots, \omega_{n}\right)$ is an orthogonal projection from the space $E_{0}=\mathcal{V} \times \mathcal{H}$ to the subspace spanned by $\left\{P_{1}(\tau), P_{2}(\tau), \cdots, P_{n}(\tau)\right\}$.

For a given time $\tau$, let $\phi_{j}(\tau)=\left\{\xi_{j}(\tau), \zeta_{j}(\tau)\right\}, j=1,2, \cdots, n .\left\{\phi_{j}(\tau)\right\}_{j=1,2, \cdots, n}$ is the standard orthogonal basis of the space $Q_{n}(\tau)_{E_{0}}=\operatorname{span}\left[P_{1}(\tau), P_{2}(\tau), \cdots, P_{n}(\tau)\right]$.

From the above, we have

$$
\begin{aligned}
\operatorname{Tr} F_{t}(\Psi(\tau)) \cdot Q_{n}(\tau) & =\sum_{j=1}^{\infty}\left(F_{t}(\Psi(\tau)) \cdot Q_{n}(\tau) \phi_{j}(\tau), \phi_{j}(\tau)\right)_{E_{0}} \\
& =\sum_{j=1}^{n}\left(F_{t}(\Psi(\tau)) \phi_{j}(\tau), \phi_{j}(\tau)\right)_{E_{0}},
\end{aligned}
$$

where $(\cdot, \cdot)_{E_{0}}$ is the inner product in $E_{0}$. Then $(\{\xi, \zeta\},\{\bar{\xi}, \bar{\zeta}\})_{E_{0}}=(\xi, \bar{\xi})+(\zeta, \bar{\zeta})$; $\left(F_{t}(\Psi) \phi_{j}, \phi_{j}\right)_{E_{0}}=-\left(\Lambda_{\varepsilon} \phi_{j}, \phi_{j}\right)_{E_{0}}-\left(g_{t}(u) \xi_{j}, \xi_{j}\right)$.

$$
\begin{aligned}
\left(\Lambda_{\varepsilon} \phi_{j}, \phi_{j}\right) & =\varepsilon\left\|\xi_{j}\right\|^{2}+\left(\varepsilon^{2}-1\right)\left(\xi_{j}, \zeta_{j}\right)+\left(\left\|A^{\frac{m}{2}} u\right\|^{2 q}-\varepsilon\right)\left(A^{m} \xi_{j}, \zeta_{j}\right)+\left(A^{m} \zeta_{j}, \zeta_{j}\right)-\varepsilon\left\|\zeta_{j}\right\|^{2} \\
& =\varepsilon\left\|\xi_{j}\right\|^{2}+\left(\varepsilon^{2}-1\right)\left(\xi_{j}, \zeta_{j}\right)+\lambda_{j}\left(\left\|A^{\frac{m}{2}} u\right\|^{2 q}-\varepsilon\right)\left(\xi_{j}, \zeta_{j}\right)+\lambda_{j}\left\|\zeta_{j}\right\|^{2}-\varepsilon\left\|\zeta_{j}\right\|^{2} \\
& \geq a\left(\left\|\xi_{j}\right\|^{2}+\left\|\zeta_{j}\right\|^{2}\right)
\end{aligned}
$$


where

$$
a:=\min \left\{\frac{2 \varepsilon+\left[1-\varepsilon^{2}+\left(\varepsilon-\left\|A^{\frac{m}{2}} u\right\|^{2 q}\right) \lambda_{j}\right]}{2}, \frac{2\left(\lambda_{j}-\varepsilon\right)+\left[1-\varepsilon^{2}+\left(\varepsilon-\left\|A^{\frac{m}{2}} u\right\|^{2 q}\right) \lambda_{j}\right]}{2}\right\}
$$

Now, suppose that $\left\{u_{0}, u_{1}\right\} \in \mathcal{A}$, according to theorem 3.3, $\mathcal{A}$ is a bounded absorbing set in $E_{1} . \Psi(t)=\left\{u(t), u_{t}(t)+\varepsilon u(t)\right\} \in E_{1}, u(t) \in D(A) ; D(A)=\{u \in \mathcal{V}, A u \in \mathcal{H}\}$.

Then there is a $s \in[0,1]$ to make the mapping $g_{t}: D(A) \rightarrow \rho\left(\mathcal{V}_{s}, \mathcal{H}\right)$. At the same time, there are the following results:

$$
\begin{aligned}
& R_{A}=\sup _{\{\xi, \zeta\} \in \mathcal{A}}|A \xi|<\infty ; \\
& \sup _{\substack{u \in D(A) \\
|A u|<R_{A}}}\left|g_{t}(u)\right|_{\rho\left(v_{S}, \mathcal{H}\right)} \leq r<\infty
\end{aligned}
$$

where $\left\|g_{t}(u) \xi_{j}, \zeta_{j}\right\|$ meets: $\left\|g_{t}(u) \xi_{j}, \zeta_{j}\right\| \leq r\left\|\xi_{j}\right\|_{s}\left\|\zeta_{j}\right\|$. Comprehensive above can be obtained:

$$
\begin{aligned}
\left(F_{t}(\Psi) \phi_{j}, \phi_{j}\right)_{E_{0}} & \leq-a\left(\left\|\xi_{j}\right\|^{2}+\left\|\zeta_{j}\right\|^{2}\right)+r\left\|\xi_{j}\right\|\left\|_{s}\right\| \zeta_{j} \| . \\
& \leq-\frac{a}{2}\left(\left\|\xi_{j}\right\|^{2}+\left\|\zeta_{j}\right\|^{2}\right)+\frac{r^{2}}{2 a}\left\|\xi_{j}\right\|_{s}^{2} .
\end{aligned}
$$

$\left\|\xi_{j}\right\|^{2}+\left\|\zeta_{j}\right\|^{2}=\left\|\phi_{j}\right\|_{E_{0}}^{2}=1$, due to $\left\{\phi_{j}(\tau)\right\}_{j=1,2, \cdots, n}$ is a standard orthogonal basis in $Q_{n}(\tau)_{E_{0}}$. So

$$
\sum_{j=1}^{n}\left(F_{t}(\Psi(\tau)) \phi_{j}(\tau), \phi_{j}(\tau)\right)_{E_{0}} \leq-\frac{n a}{2}+\frac{r^{2}}{2 a}\left\|\xi_{j}\right\|_{s}^{2} .
$$

Almost to all $t$, making

$$
\sum_{j=1}^{n}\left\|\xi_{j}\right\|_{s}^{2} \leq \sum_{j=1}^{n-1} \lambda_{j}^{s-1}
$$

So

$$
\operatorname{TrF}_{t}(\Psi(\tau)) \cdot Q_{n}(\tau) \leq-\frac{n a}{2}+\frac{r^{2}}{2 a} \sum_{j=1}^{n-1} \lambda_{j}^{s-1} .
$$

Let us assume that $\left\{u_{0}, u_{1}\right\} \in \mathcal{A}$, is equivalent to $\Psi_{0}=\left\{u_{0}, u_{1}+\varepsilon u_{0}\right\} \in R_{\varepsilon} \mathcal{A}$. Then

$$
\begin{aligned}
& q_{n}(t)=\sup _{\Psi_{0} \in R_{\varepsilon} \mathcal{A} \mathcal{A}} \sup _{\substack{\omega \omega E_{0} \\
\|\omega\|_{0} \leq 1}}\left(\frac{\int_{0}^{t} \operatorname{Tr} F_{t}\left(S_{\varepsilon}(\tau) \Psi_{0}\right) \cdot Q_{n}(\tau) \mathrm{d} \tau}{t_{0}}\right), j=1,2, \cdots, n, \\
& q_{n}=\limsup _{t \rightarrow \infty} q_{n}(t) .
\end{aligned}
$$

According to (4.19), (4.20), so

$$
\begin{aligned}
& q_{n}(t) \leq-\frac{n a}{2}+\frac{r^{2}}{2 a} \sum_{j=1}^{n-1} \lambda_{j}^{s-1}, \\
& q_{n} \leq-\frac{n a}{2}+\frac{r^{2}}{2 a} \sum_{j=1}^{n-1} \lambda_{j}^{s-1} .
\end{aligned}
$$


Therefore, the Lyapunov exponent of $\mathcal{A}$ (or $R_{\varepsilon} \mathcal{A}$ ) is uniformly bounded.

$$
\mu_{1}+\mu_{2}+\cdots+\mu_{n} \leq-\frac{n a}{2}+\frac{r^{2}}{2 a} \sum_{j=1}^{n} \lambda_{j}^{s-1}
$$

From what has been discussed above, it exists $n \geq 1$, a and $\mathrm{r}$ are constants, then

$$
\begin{gathered}
\frac{1}{n} \sum_{j=1}^{n} \lambda_{j}^{s-1} \leq \frac{a^{2}}{6 r^{2}}, \\
q_{n} \leq-\frac{n a}{2}\left(1-\frac{r^{2}}{a^{2}} \sum_{j=1}^{n} \lambda_{j}^{s-1}\right) \leq-\frac{5 n a}{12}, \\
\left(q_{j}\right)_{+} \leq \frac{r^{2}}{2 a} \sum_{i=1}^{j} \lambda_{i}^{s-1} \leq \frac{n a}{12}, j=1,2, \cdots, n, \\
\max _{1 \leq j \leq n-1} \frac{\left(q_{j}\right)_{+}}{\left|q_{n}\right|} \leq \frac{1}{5} .
\end{gathered}
$$

According to the reference [6] [7], we immediately to the Hausdorff dimension and fractal dimension are respectively $d_{H}(\mathcal{A}) \leq \frac{n}{5}, d_{F}(\mathcal{A}) \leq \frac{6 n}{5}$.

\section{Conclusion}

In this paper, we prove that the higher-order nonlinear Kirchhoff equation with linear damping in $L^{\infty}\left((0,+\infty) ; H^{2 m}(\Omega) \times H_{0}^{m}(\Omega)\right)$ has a unique smooth solution $\left(u, u_{t}\right)$. Further, we obtain the solution semigroup $S(t): H^{2 m}(\Omega) \times H_{0}^{m}(\Omega) \rightarrow H^{2 m}(\Omega) \times H_{0}^{m}(\Omega)$ has global attractor $\mathcal{A}$. Finally, we prove the equation has finite Hausdorff dimensions and Fractal dimensions in $L^{\infty}\left((0,+\infty) ; H^{2 m}(\Omega) \times H_{0}^{m}(\Omega)\right)$.

\section{Acknowledgements}

The authors express their sincere thanks to the aonymous reviewer for his/her careful reading of the paper, giving valuable comments and suggestions. These contributions greatly improved the paper.

\section{Fund}

This work is supported by the National Natural Sciences Foundation of People's Republic of China under Grant 11561076.

\section{References}

[1] Ghisi, M. and Gobbino, M. (2009) Spectral Gap Global Solutions for Degenerate Kirchhoff Equations. Nonlinear Analysis, 71, 4115-4124. https://doi.org/10.1016/j.na.2009.02.090

[2] Yang, Z.J., Ding, P.Y. and Li, L. (2016) Longtime Dynamics of the Kirchhoff Equations with Fractional Damping and Supercritical Nonlinearity. Journal of Mathematical Analysis Application, 442, 485-510. https://doi.org/10.1016/j.jmaa.2016.04.079

[3] Yang, Z.J., Ding, P.Y. and Liu, Z.M. (2014) Global Attractor for the Kirchhoff Type Equations with Strong Nonlinear Damping and Supercritical Nonlinearity. Applied Mathematics Letters, 33, 12-17. https://doi.org/10.1016/j.aml.2014.02.014 
[4] Li, F.C. (2004) Global Existence and Blow-Up of Solutions for a Higher-Order KirchhoffType Equation with Nonlinear Dissipation. Applied Mathematics Letters, 17, 1409-1414. https://doi.org/10.1016/j.am1.2003.07.014

[5] Li, Y. (2011) The Asymptotic Behavior of Solutions for a Nonlinear Higher Order Kirchhoff Type Equation. Journal of Southwest China Normal University, 36, 24-27.

[6] Teman, R. (1998) Infinite Dimensional Dynamics Systems in Mechanics and Physics. Springer, New York.

[7] Wu, J.Z. and Lin, G.G. (2009) The Global Attractor of the Bossinesq Equation with Damping Term and Its Dimension Estimation. Journal of Yunnan University, 31, 335-340.

[8] Yang, Z.J. (2007) Longtime Behavior of the Kirchhoff Type Equation with Strong Damping on $R_{N}$. Journal of Differential Equations, 242, 269-286. https://doi.org/10.1016/j.jde.2007.08.004

[9] Yang, Z.J. and Liu, Z.M. (2015) Exponential Attractor for the Kirchhoff Equations with Strong Nonlinear Damping and Supercritical Nonlinearity. Applied Mathematics Letters, 46, 127-132. https://doi.org/10.1016/j.aml.2015.02.019

[10] Lin, G.G. (2011) Nonlinear Evolution Equation. Yunnan University Press, Kunming.

\section{Submit or recommend next manuscript to SCIRP and we will provide best service} for you:

Accepting pre-submission inquiries through Email, Facebook, LinkedIn, Twitter, etc.

A wide selection of journals (inclusive of 9 subjects, more than 200 journals)

Providing 24-hour high-quality service

User-friendly online submission system

Fair and swift peer-review system

Efficient typesetting and proofreading procedure

Display of the result of downloads and visits, as well as the number of cited articles

Maximum dissemination of your research work

Submit your manuscript at: http://papersubmission.scirp.org/

Or contactijmnta@scirp.org 